\title{
Analysis of Factors Affecting Multimedia Delivery for Elderly People
}

\author{
Prissadang Suta, Naovarat Limdumrongnukoon, Panupong Chalardkitsirikul, \\ Thaspoom Suntiyotin, Pornchai Mongkolnam ${ }^{\mathrm{a}, *}$, and Jonathan Hoyin Chan ${ }^{\mathrm{b}, *}$ \\ School of Information Technology, King Mongkut's University of Technology Thonburi, Thungkru, \\ Bangkok 10140, Thailand \\ E-mail: ${ }^{2}$ pornchai@sit.kmutt.ac.th, bjonathan@sit.kmutt.ac.th (Corresponding authors)
}

\begin{abstract}
This study aims to apply appropriate information technology services for elderly people in order to fill the communication gap between the elderly, family members and friends by using a multimedia delivery platform. The aim is to improve the quality of life of the elderly people who live alone. It is intended to enhance the lives of elderly people to stay connected with family members, and friends by sending multimedia content including text, audio, image and video. We analyze the factors affecting the elderly person's intention to use the platform by using Unified Theory of Acceptance and Use of Technology (UTAUT) model. The model evaluates the effectiveness of the multimedia delivery platform over the six key UTAUT constructs, namely, Performance Expectancy, Effort Expectancy, Social Influence, Facilitation Conditions, Perceived Security, and Experience and Voluntariness to Behavioral Intention. The analysis is twofold: to determine the factors affecting the intention of the elderly people in using the multimedia delivery platform, and to investigate how to reduce the communication gap between the elderly, family members and friends.
\end{abstract}

Keywords: UTAUT model, multimedia delivery platform, elderly people, quality of life.

ENGINEERING JOURNAL Volume 22 Issue 1

Received 15 June 2017

Accepted 31 August 2017

Published 31 January 2018

Online at http://www.engj.org/

DOI:10.4186/ej.2018.22.1.49 


\section{Introduction}

World's elderly population is increasing in an alarmingly rapid pace. The World Population Ageing 2015 [1] reported and projected ageing population trends between 2000 and 2050. In 2015, there were 901 million elderly people aged 60 years or more, which was an increase of 48\% from 607 million in 2000, and it was projected to increase by $56 \%$ in 2030 with 1,402 million elderly people. By 2050 , the global ageing population was projected to grow to nearly 2,092 million elderly people. In Thailand, the percentage of ageing population aged 60 years or over is expected to increase from $12.89 \%$ (8.5 million) in 2010 to $19.12 \%$ (12.62 million) in 2020 and is projected to rise further to $26.56 \%$ (17.58 million) in 2030 [2]. Although most elderly people in Thailand live together in a family, we found the trend of the elderly living alone had increased from $6.3 \%$ in 2002 to $10.4 \%$ in 2014, according to the 2014 survey of the older persons in Thailand [3]. Major problems of the elderly who live alone are loneliness and isolation, having no one at home to take care of, and needing to work to support daily living. Now the elderly people tend to live longer and alone in large part due to a healthcare advancement and structural social changes. This causes a great deal of concerns in terms of loneliness, social isolation, and depression among the elderly. Moreover, it is found that the main daily activity of the elderly living alone at home is spending time around four to six hours with television. Reid [4] presented that the TV's roles in the lives of the elderly as an important window to the world. It provided various types of programs that met the elderly people's needs. The elderly received up-to-date news and useful information. As a result, they were more involved, entertained, acquiring information and knowing world affairs and issues, and, of course, passing time.

We previously proposed a conceptual model for delivering multimedia by using various sources to the elderly people who lived alone at home via television, with the intention of improving their quality of life [5]. The elderly might have a problem remembering something, such as forgetting to take medications or miss an appointment with the doctor. We consequently applied information technology for the elderly in order to fill in communication gaps between the elderly, family members and friends. We designed the prototype to help the elderly stay active and in touch with their family members and friends [6-7]. The user interface was simple with colors suitable for the elderly. The multimedia delivery platform, called Argong which means grandfather in Thai, comprised television, set-top box and its application, a remote control application running on a tablet, server and database. The set-top box was connected with smart TV or Android television, and the tablet computer was used as a remote control. The system aimed to deliver multimedia from all types of source devices such as smartphones, tablets and personal computers to the destined home television of the elderly. It was intended to strengthen family relationships in order to reduce loneliness and social isolation. Family members and friends could send activity reminders and encourage social interactions with the elderly. The system could bring up and show past multimedia from family members and friends that had been sent to the elderly at a scheduled interval to keep the elderly connected. The multimedia contents could be text, photo, video and audio. Furthermore, the elderly could control the TV with a touchscreen on the tablet, as well as be able to use other similar functions found in a traditional television remote control. In this continued study, we propose an evaluation of effectiveness of the multimedia delivery platform by using Unified Theory of Acceptance and Use of Technology (UTAUT) model. It provides a model to predict the elderly behavior intention to accept and use the Argong multimedia delivery platform.

In the next few sections, we provide literature reviews, materials and methods. The materials describe the key components of the Argong system, followed by the prototype, system implementation, and system composition designed for the elderly people. The method describes participants, procedure and hypothesis testing. Last, we describe the data analysis, results, conclusions and future work.

\section{Literature Review}

There is an increasing interest in a multimedia communication delivery [8-9] for the elderly such as medreminder application reminded people to take medicines, and call relatives or medical personnel in case of emergency through an interactive television [10]. The Senior Channel system allowed older users to interact with TV and multimedia contents, which in turn helped to improve their social inclusion and participation [11]. A control service framework, termed Multi-user Variable Remote Control iTV (MVC-iTV) service, was developed and it focused on the integration of large-scale mobile and convenient remote controls for multiuser interaction with family within a home information system [12]. Users could use their own handheld devices to interact with the iTV service via internet communications. The SAID project was developed and 
aimed to provide the elderly people with more efficient social care and service improvement over TV for delivering a complete catalogue of services by combining with the iTV technologies and intelligent agents in their home environment [13]. Remote health monitoring was used to interact between the user and system by using a digital television connected with a set-top box to receive the patient's data collection through a properly interfaced network of biological sensors such as a blood pressure, temperature monitor and an accelerometer for movement analysis [14]. A home server platform integrated a home multimedia server, a home control server and a home information server and was used to help manage digital information appliances like internet refrigerators and microwave ovens, so the user could access all of devices connected with the home server [15]. An interaction between a wired home network and a wireless communication network was used to control consumer electronics devices such as TV, VCR, camera, CD player, and radio by using a home audio video interoperability (HAVi) and wireless application protocol (WAP) [16]. A TVHealth prototype assisted caregivers or patients in home care [17].

Currently, more research focuses on how to have a good care for the elderly and improve the relationship between the elderly and their family members. Ideas to use information technology to help elderly people have long been proposed, and there exist some applications on smartphones such as MedCoach to remind the elderly to take a pill, or a Red Panic Button to provide help to the elderly when accidents occur [18]. However, there are some limitations for creating an application to help the elderly because many of them do not use smartphones. Thus, these smartphone applications cannot effectively help the elderly. According to many studies, the elderly always spend most of their time watching televisions, and in some ways television is a good medium for effectively conveying information to the elderly, and because there are not many applications developed to help the elderly while they are watching television; therefore, we have developed an application for an Android box with the ability to use a tablet as a remote control.

\section{Materials \& Methods}

\subsection{Prototypes}

The Argong multimedia delivery platform for the elderly is divided into two parts, which are Argong TV and Argong remote control. First, the Argong TV application needs to be installed on the Android set-top box or Android TV. When the TV is turned on, a home screen launcher will be shown as in Fig. 1(a), and the Argong TV application will be automatically launched without any further needed input from the user. Second, the Argong remote control needs to be installed on the tablet computer. Figure 1(b) shows its home screen. Tablet computer is a portable device which is easy to use as a touchscreen and easy to see with a wide range of screen resolutions and sizes, and thus it is most suitable for the elderly users. The Argong remote control can connect to the TV by logging on to the same user account.

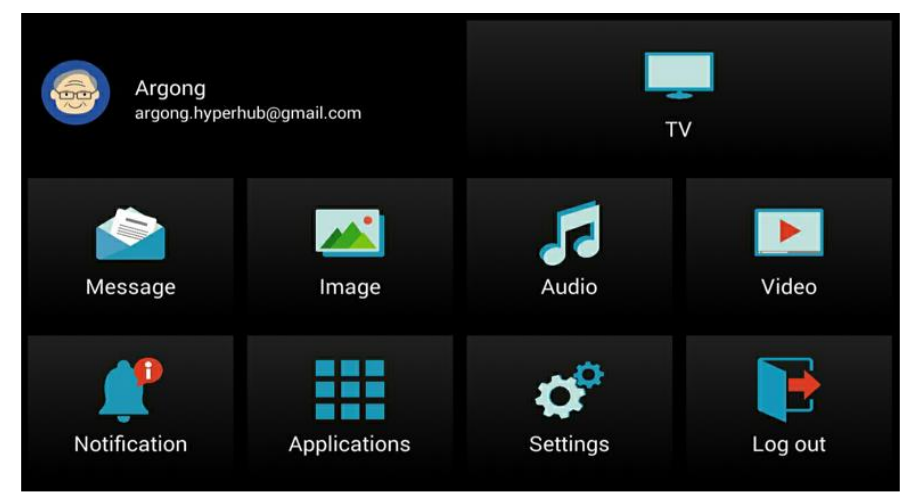

(a) 


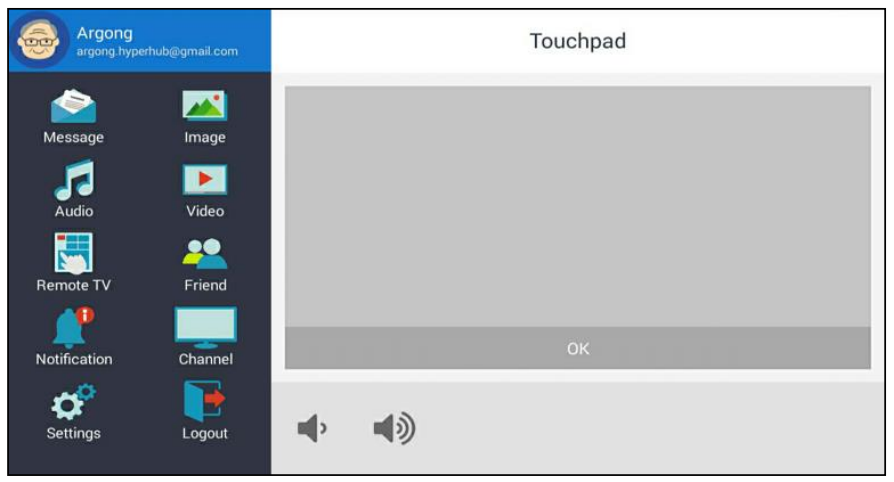

(b)

Fig. 1. Home screen of Argong multimedia delivery platform: (a) Argong TV application; and (b) Argong remote control application.

\subsubsection{System implementation}

The Argong multimedia delivery platform consists of the set-top box which is connected to a smart TV or Android TV, and the tablet computer which acts as a remote control and controls some TV functions via infrared (IR), as shown in Fig. 2.

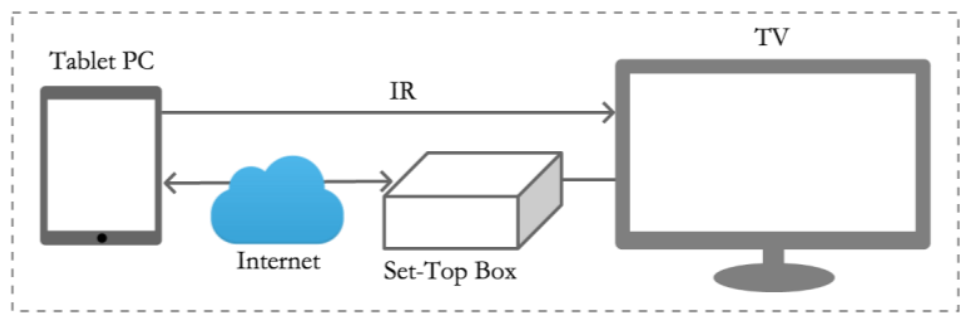

Fig. 2. The Argong multimedia delivery platform.

The Argong application is developed on Android based on the Java programming language. The proposed system includes the following components.

- Hardware: LED/LCD TV, Android set-top box, Android tablet computer

- Software: Android Studio, MySQL Server, Firebase

\subsubsection{System composition}

The Argong application comprises three components, which are multimedia delivery platform, application server including a server and database, and Firebase used for receiving push notifications from the server, as shown in Fig. 3. The five key elements of the Argong application are TV channel, Message, Reminder, Touchpad, and Remote Control, and all are embedded in the device. Further details of these elements are as follows. 


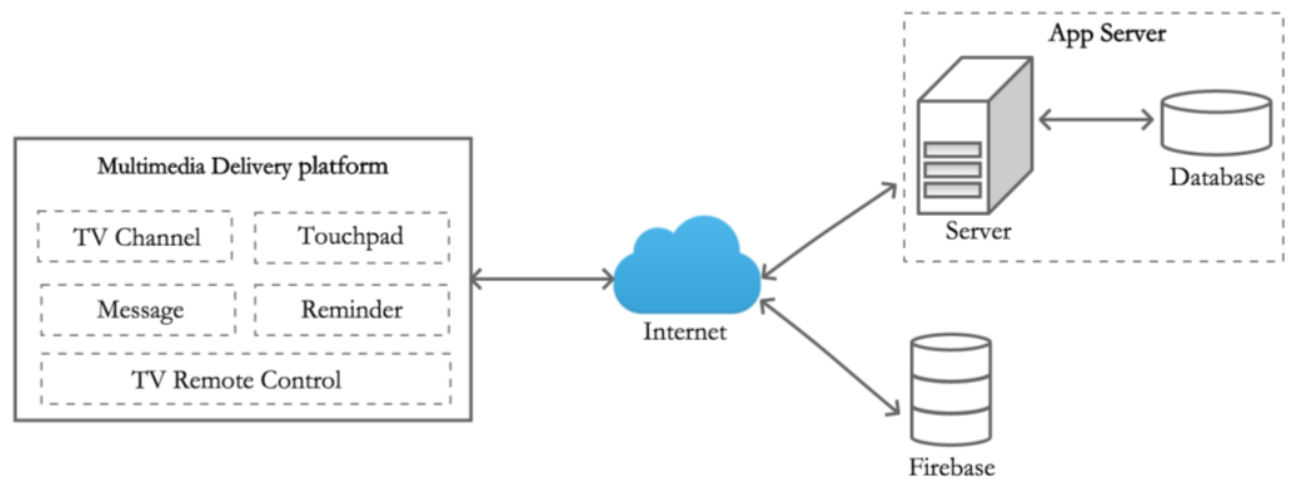

Fig. 3. The Argong application architecture.

1) TV Channel: It is a live streaming format which is an extension to the m3u format encoded in UTF8 characters. The Internet Protocol Television (IPTV) provides digital television services over Internet Protocol (IP) to broadcast ordinary TV programs [19].

2) Message: The elderly can send multimedia contents to family members and friends by using an Android tablet PC. The delivery mean is like an email form, including sender, receiver, subject heading, and multimedia contents. It masks a folder as unread and read messages accordingly, as well as the number of messages. The multimedia contents include the following.

- Text: The elderly can use a virtual keyboard or a speech-to-text function on a tablet computer for typing messages, and then send the messages to family members and friends. When typing messages, the system enables a word prediction function to auto complete the frequently used words or phrases. This function facilitates the elderly to type effectively, easily and conveniently. For the speech-to-text feature, we use RecognizerIntent class [20], an Android speech-to-text engine, for converting speeches to texts. The elderly simply speaks the messages, and they will be converted as texts which are accurate and correct contextually. The system simply prompts the user for speeches and send them through the speech recognizer, and then message will be sent back and displayed as texts. It supports both Thai and English languages.

- Picture, Audio, and Video: The elderly can choose existing pictures, audios or videos from a gallery stored on the tablet computer, or take a new picture, audio or video. The system allows for previewing the contents before the elderly send them to family members or friends. File Transfer Protocol (FTP) is a standard Internet protocol for transmitting files between computers on the Internet over TCP/IP connections, which is used to upload multimedia file to the server; it runs on a background thread and will be disconnected after transferring files successfully.

3) Reminder: The system can notify scheduled reminders or special events, such as taking medicines, paying a bill or meeting when the appointment time is approaching. The elderly can add the reminders themselves, which specify four trigger types, including one time: the message notifies once, daily: the message notifies at the same time every day, weekly: the message notifies at the same time each week, and monthly: the message notifies at the same time each month. Family members can also send the activity reminders to their elderly parents. Furthermore, the system will alert the elderly with new messages on both TV and tablet computer screen when the messages arrive. If the elderly clicks a view button on the prompt new message while watching, the TV will switch to the Argong TV launcher and show the message.

4) Touchpad: The elderly can use the touchpad to control a cursor on the television screen instead of using a traditional television remote control. Lai and Hwang [21] proposed the usability evaluation of three virtual pointing models including virtual touchpad, virtual joystick and virtual direction key. Their results showed that the virtual touchpad took the least time to select the target object, and users were satisfied with the virtual touchpad; therefore, we decide to use the virtual touchpad to control the cursor on television for our system. We use the Android getevent and sendevent commands for developing this feature [22-24]. The getevent command runs on the device and 
provides information about input devices and a live dump of kernel input events from the input devices which are connected to the Android set-top box. It splits out the input events in a hexadecimal format. Moreover, we use Android motion events which report the movement that helps to detect the gestures when the user's finger touches and moves the cursor on the TV screen [25]. The system gets the touch data such as the coordinates $\mathrm{x}$ and $\mathrm{y}$. The sendevent command is used to inject the input events to the input device, which allows the elderly to move a cursor around the TV screen. The command format is as follows:

\section{[command] [device] [type] [code] [value]}

The parameters are defined as follows:

- command - sendevent

- device - dev/input/eventX; $\mathrm{X}$ is a number; the input device name is stored as string type. We find what input device name works for touch event which uses the getevent command. The number of touch data will be event 0 to event 9 .

- type: It is the touch event type for generating events which is stored as a decimal type.

- code: It is the sequence of input events. The event code is stored as a decimal type.

- value: The value is stored as a decimal type.

Based on Pointing task performance, Cheong et al. [26] used three representative devices for remote pointing devices in order to compare them to a standard Mouse 2.0 for the performance evaluation. First, the optical finger navigation $(\mathrm{OFN})$; it had a touchpad which allowed a freely movement with 2D navigation. Second, the Hall mouse; it had four Hall sensors recognizing the movement directions. Last, the GyroPoint; a user could control a pointer by a wrist movement. The results of the pointing devices depended on the tasks of different performances referred to the Fitts' Law [27] specified in ISO 9241-9 measurement. For a steering tunnel task on a curved trail, the Hall mouse and OFN showed a higher accuracy than the GyroPoint; therefore, we chose remote pointing devices working similar to the Hall mouse and OFN to implement the Argong system. MacKenzie [28] showed the experiment on a touch-based target selection by calculating the Fitts' Law and throughput, which were compared between direct input as a finger and indirect input as a mouse, the result is a performance enhancement for touch by about 50 percent than accepted values for a mouse. A user could choose the direct input, which did not require the user to track pointing symbol and to press the button. The main movement-based tasks in a graphical user interface were targeting, tracking, and targeted-tracking using a mouse, a pen mouse, a touch screen, and a graphics tablet [29].

We run an experiment to compare between the Argong touchpad and a traditional television remote control in pointing task by using the concept of Fitts' Law to model the pointing performance which was based on the size and distance of the target object as in Eq. (1).

$$
M T=a+b \log _{2}\left(2 \frac{A}{W}\right)
$$

It included a movement time (MT), constants (a, b), target amplitude (A) and target width (W). We used the FittsTouch application [30] to do the experiment that conformed to ISO 9241 Part 9 standards [31] which was about requirements for non-keyboard input devices. We evaluated the system by using TV: LCD TV LG M2341A, Android set-top box: Tronsmart Draco AW80 with Android 4.4 (KitKat), and Android tablet computer: Samsung Galaxy Note 10.1 N8000 with Android 4.4 (KitKat). All 30 elderly users tested this feature on the same devices. The experiment was set with the following parameters:

- The task type was two-dimensional (2D) movements, with the horizontal axis and the vertical axis.

- The target diameters were 250 and 500 pixels.

- The target widths were 60 and 100 pixels. 
Thereafter, the performance evaluation was calculated by using a paired t-test. It measured the same subjects by comparing differences in the use of a touchpad and a remote control. The study proposed the following hypothesis:

- $\mathrm{H}_{0}: \mu_{1}=\mu_{2}$; the population mean of the differences $\left(\mu_{1}\right)$ equalled the hypothesized mean of the differences $\left(\mu_{2}\right)$.

- $H_{1}: \mu_{1} \neq \mu_{2}$; the population mean of the differences $\left(\mu_{1}\right)$ did not equal the hypothesized mean of the differences $\left(\mu_{2}\right)$.

We found the confidence interval at 95\% confidence level and p-value equal to 0 . The correlation between the indices of difficulty (IDe) and movement time (MT) is shown in Fig. 4(a). The touchpad has a lower movement time than remote control. Figure 4(b) shows the average of using performance of the touchpad at 0.44 , which is higher than the remote control at 0.35 .

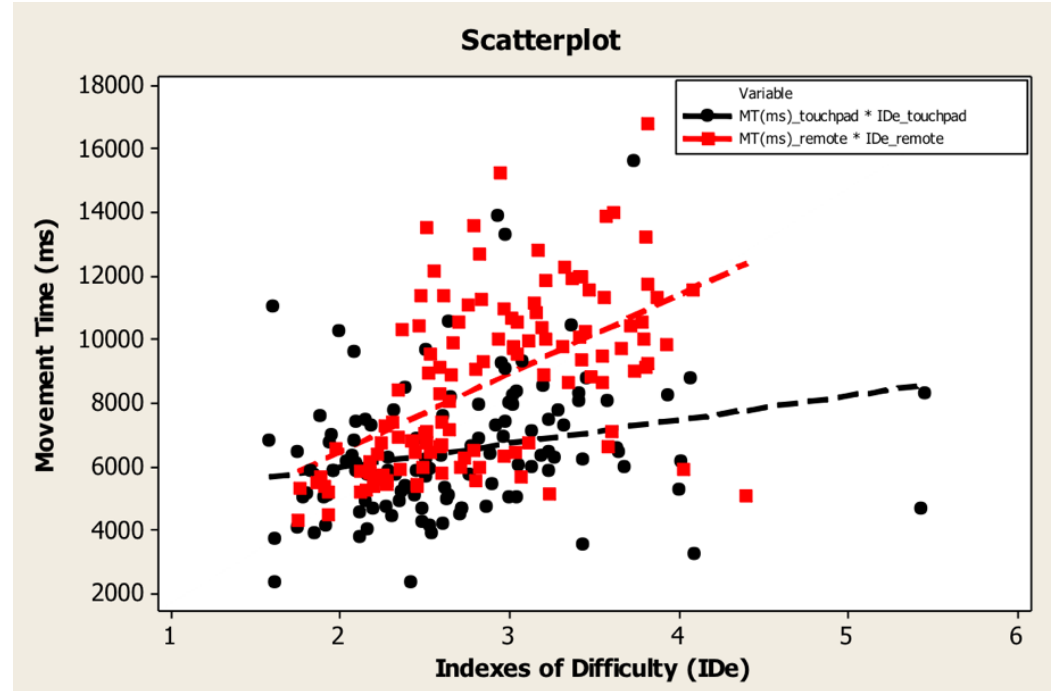

(a)

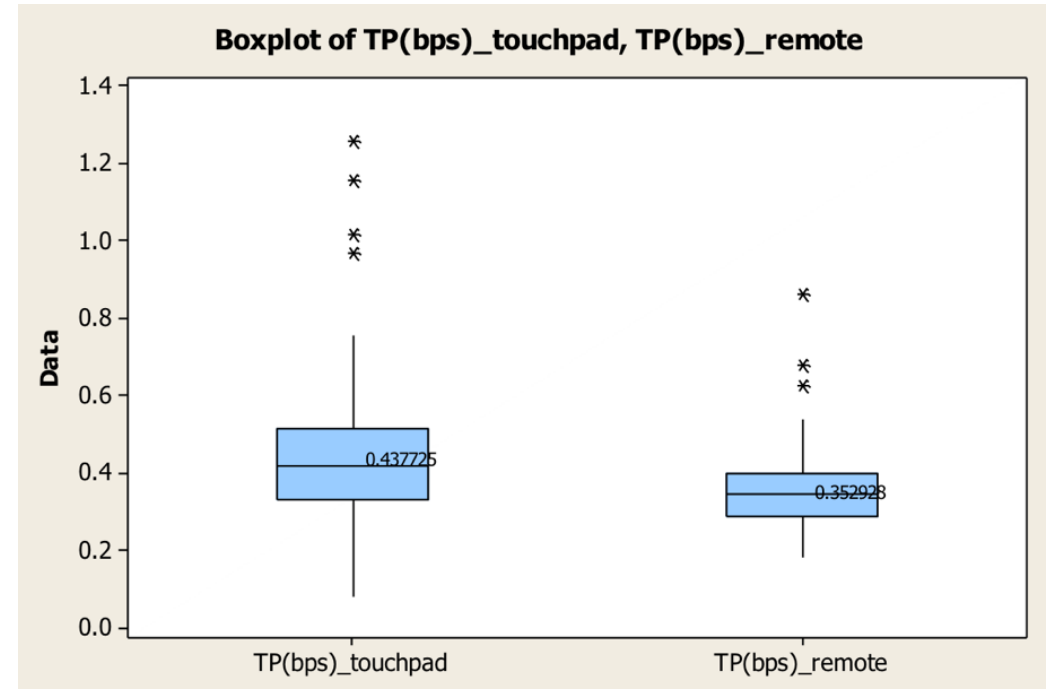

(b)

Fig. 4. Performance comparisons of the indexes of difficulty and movement time.

5) Remote Control: The elderly can control TV functions by using the tablet computer that has a builtin infrared blaster which works similarly to the traditional television remote control, such as power on/off, mute/un-mute, volume up/down, channel up/down, menu bottom with up/down/ and left/right controls, high-definition multimedia interface (HDMI) mode, TV mode and source menu. We use ConsumerIrManager, an Android's infrared (IR) transmitter API, which operates a consumer 
infrared on a device [32]. The system starts with IR codes prepared in the Pronto hex format which is based on the device brands such as Samsung, Sony, Toshiba and LG and convert its number to a decimal format using hex $2 \mathrm{dec}$ function. The first number of IR codes is the frequency of the IR carrier in terms of the Pronto internal clock, and the other numbers are a count pattern. Frequency is measured in cycles or pulses per second. The count pattern is converted to the duration pattern by multiplying each value of the pulses. Finally, the system transmits the infrared pattern.

\subsection{Methods}

According to the Unified Theory of Acceptance and Use of Technology (UTAUT) [33], researchers were likely to use it for a user acceptance prediction, such as a conceptual model of effect and behavior among the elderly, based on social environmental factors, on a patient portal adoption that consists of three institutional forces: normative, mimetic, and coercive. It was built on institutional theory and UTAUT. Cimperman M. et al. [34] proposed a model at a conceptual level which measured constructs in the UTAUT model for predicting the factors affecting older users' acceptance of Home Telehealth Services (HTS), which were information and communication technology based services using Internet connections and computers or mobile devices in a conservative field of healthcare. We also found several works that used and applied the UTAUT model for a user intention to accept a technology [35-36], applied the UTAUT model for students at Methodist University College Ghana for their intentions to use information and communication technology (ICT) available for learning and research. For this study, we also apply the UTAUT model with the following two main goals: to determine the factors affecting the elderly people intention to use the multimedia delivery platform, and to improve the communication gap between the elderly, family members and friends by using the multimedia delivery platform.

To achieve the two goals, we asked 30 elderly persons to use our Argong system and respond to a questionnaire as shown in Fig. 5. The details are given as follows.

\subsubsection{Participants}

Thirty elderly people aged 60 years or over who live in Thailand. The sample size of 30 is considered typically large enough for repeatedly sampled means to be approximately normally distributed [37].

\subsubsection{Procedure}

We described the goals of this study and explained experimental procedures to the elderly before they started testing the Argong system. We designed the procedures as follows:

1) The elderly tested the Argong remote control features that worked similarly to a traditional TV remote control, such as power on/off, mute/un-mute, volume up/down, channel up/down, menu bottom with up/down/ and left/right controls, HDMI mode, TV mode and source menu.

2) The elderly tested a touchpad feature to control a cursor movement on the television screen.

3) The elderly sent multimedia contents to family members or friends by using the tablet with the Argong remote control application. First, the elderly selected multimedia type menu, including message, image, audio and video to be sent to family members or friends. Next, the elderly selected one or more receivers from the receiving family and friend list. The steps taken of each menu are as follows:

- Message menu: The elderly typed messages or used a speech-to-text feature. Next, the elderly clicked a send button.

- Image menu: The elderly took a new picture, and then clicked a send button. Next, the elderly selected a picture from the gallery, and then clicked the send button.

- Audio menu: The elderly recorded a new audio, and then clicked a send button. Next, the elderly selected the audio from the music track or sound picker, and clicked the send button. However, the elderly could listen to the selected audio before sending it out. 
- Video menu: The elderly recorded a new video, and then clicked a send button. Next, the elderly selected the video from the gallery, and then clicked the send button. However, the elderly could preview the selected video before sending it out.

4) The elderly received multimedia contents via television with the Argong application. First, the elderly clicked the TV menu. The system showed live channels. The elderly slid the left menu which showed sub menus such as unread message, read message, and sent message. When elderly selected unread message menu, then the system showed a list of new messages whose all types of multimedia contents were listed. The elderly selected a new message, and then the system popped up the window of message details. Next, the message was moved to the read message menu after the elderly closed the message window. The elderly could open messages to read again via the TV home screen menu.

5) The elderly scheduled the upcoming reminders or special events. First the elderly selected notification menu. Next the elderly selected a trigger as one-time type, and then typed a message to alert at that time.
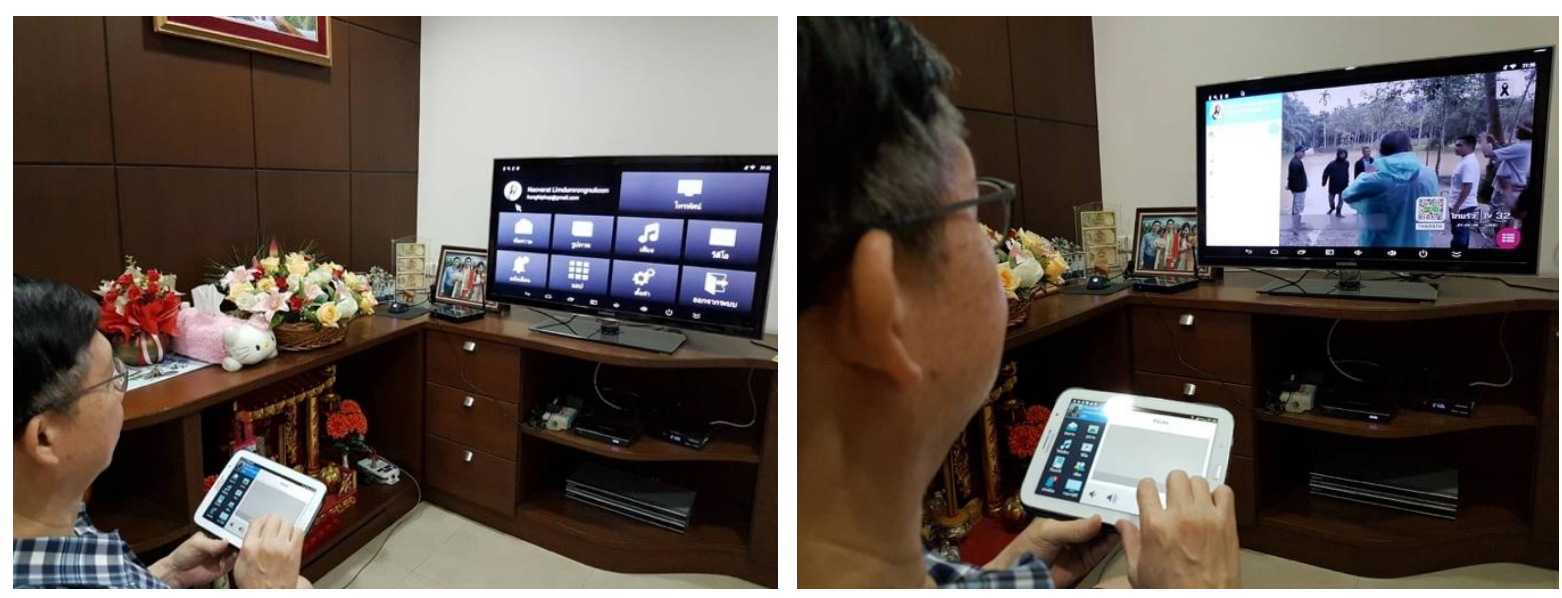

Fig. 5. An elderly person used the Argong system before doing a questionnaire.

\subsubsection{Hypothesis testing}

The survey instruments are divided into three parts as follows: the first part is general information about the elderly; the second part is survey items adopted from literature reviews of behavioural intention to use, and the third is textual comments or suggestions for improving the multimedia delivery platform. The six UTAUT constructs are Performance Expectancy, Effort Expectancy, Social Influence, Facilitation Conditions, Experience, and Voluntariness. Their definitions are as follows. Performance Expectancy is defined as the degree to which an individual believes that using the system will help him or her to attain gains in job performance. Effort Expectancy is defined as the degree of ease associated with the use of system. Social Influence is defined as the degree to which an individual perceives the importance of others' belief that he or she should use the new system. Facilitation Conditions is defined as the degree to which an individual believes that an organizational and technical infrastructure exist to support use of the system. Based on the UTAUT model we investigate the elderly intention to use the Argong system with a questionnaire by using a 5-point Likert-type scale for responses $(1=$ Very Unsatisfied, $2=$ Unsatisfied, $3=$ Moderate, $4=$ Satisfied, and $5=$ Very Satisfied). Based on the UTAUT, we propose the model as shown in Fig. 6 with the following hypotheses. 


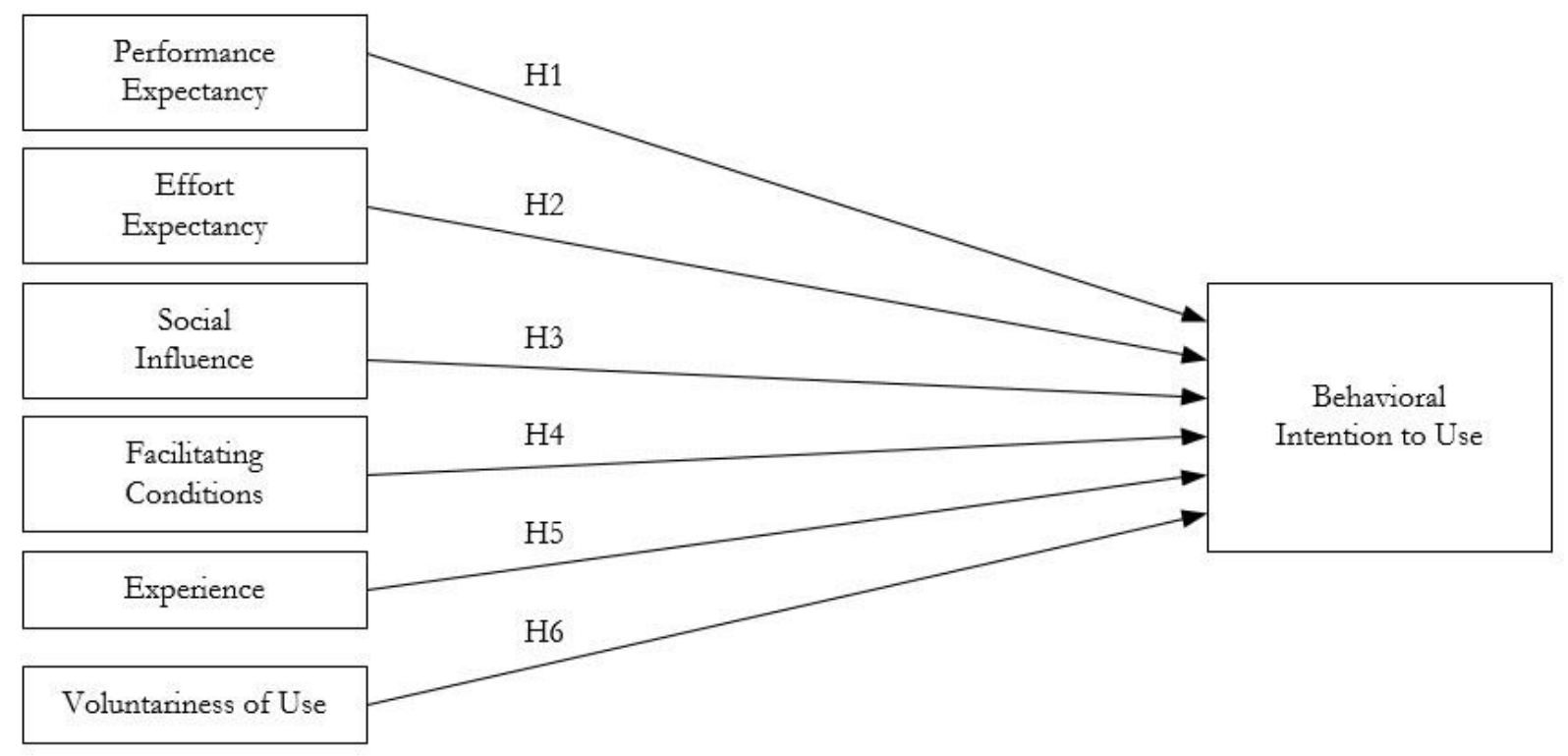

Fig. 6. Extended UTAUT model.

H1: Performance Expectancy instruments will increase a behavioural intention to use the Argong by the elderly.

H2: Effort Expectancy instruments will increase a behavioural intention to use the Argong by the elderly.

H3: Social Influence instruments will increase a behavioural intention to use the Argong by the elderly.

H4: Facilitation Conditions instruments will increase a behavioural intention to use the Argong by the elderly.

H5: Experience instruments will increase a behavioural intention to use the Argong by the elderly.

H6: Voluntariness of Use instruments will increase a behavioural intention to use the Argong by the elderly.

\section{Data analysis and Results}

\subsection{Instrument Validation}

Table 1 indicates the reliability coefficients of the constructs by using the Cronbach's alpha coefficients for the measurement model [38]. If the threshold value of the alpha coefficient is greater than 0.7, the questionnaire answer is considered a reliable acceptance. The results show that all the factors have the coefficients higher than the cutoff value 0.7 , and thus the survey instruments are reliable.

Table 1. Cronbach's alpha coefficients for the measurement model.

\begin{tabular}{lc}
\hline \multicolumn{1}{c}{ Factor } & Cronbach's Alpha Coefficient \\
\hline Performance Expectancy & 0.954 \\
Effort Expectancy & 0.756 \\
Social Influence & 0.946 \\
Facilitating Conditions & 0.950 \\
Experience & 0.743 \\
Voluntariness of Use & 0.958 \\
Behavioural Intention to Use & 0.981 \\
\hline
\end{tabular}

\subsection{Data Analysis}

According to the hypotheses presented above, the instruments were conducted with 30 elderly persons in Thailand. The elderly fully completed the survey instruments. The demographic and background 
characteristics of the respondents are shown in Table 2, with gender, age, highest level of education, experience with technology, hours of TV viewing time per day, living pattern, and home Internet access.

Table 2. Background of respondents.

\begin{tabular}{ll}
\hline Gender & - Male: $7(23.3 \%)$ \\
& - Female: $23(76.7 \%)$ \\
\hline Age & $-60-64$ years: $7(23.3 \%)$ \\
& $-65-69$ years: $8(26.7 \%)$ \\
& $-70-74$ years: $9(30 \%)$ \\
& $-75-79$ years: $4(13.3 \%)$ \\
& $-80-84$ years: $2(6.7 \%)$ \\
& -85 or older: 0 \\
\hline Highest level of education & - Less than Bachelor's degree: $19(63.3 \%)$ \\
& - Bachelor's degree: $7(23.3 \%)$ \\
& - Master's degree: $4(13.3 \%)$ \\
& - Doctoral degree: 0 \\
& - Other: 0 \\
\hline Experience with technology & - Computer: $1(3.3 \%)$ \\
& - Smart phone: $13(43.3 \%)$ \\
& - Tablet Computer: $2(6.7 \%)$ \\
& - None: $14(46.7 \%)$ \\
\hline Hours of TV viewing time per day & $-1-4$ hours: $22(73.3 \%)$ \\
& $-5-8$ hours: $5(16.7 \%)$ \\
& $-9-12$ hours: $2(6.7 \%)$ \\
& - Other: $1(3.3 \%)$ \\
\hline Living pattern & - Alone: $9(30 \%)$ \\
& - Spouse: $12(40 \%)$ \\
& - Children: $9(30 \%)$ \\
\hline Home Internet access & - Yes: $17(56.7 \%)$ \\
& - None: $13(43.3 \%)$ \\
\hline &
\end{tabular}

First, we investigated each factor that might affect the intention to use the Argong system. The statistical measures were mean and standard deviation values as shown in Table 3, which are based on the 5-point Likert-type scale of the responses.

Table 3. Survey items for estimating UTAUT model.

\begin{tabular}{lcc}
\hline \multicolumn{1}{c}{ Questionnaire } & Mean & $\begin{array}{c}\text { Standard } \\
\text { Deviation }\end{array}$ \\
\hline Performance Expectancy (PE) & 4.29 & 0.70 \\
\hline $\begin{array}{l}\text { PE1: I find that using the system would help better communicate } \\
\text { with my family members. }\end{array}$ & 4.30 & 0.79 \\
\hline $\begin{array}{l}\text { PE2: I find that using the system would strengthen family } \\
\text { relationships and bonding. }\end{array}$ & 4.20 & 1.00 \\
\hline $\begin{array}{l}\text { PE3: I find that using the system would be helpful in notifying } \\
\text { upcoming events. }\end{array}$ & 4.43 & 0.68 \\
\hline PE4: I find that using a touchpad for controlling TV is easy to use. & 4.27 & 0.74 \\
\hline PE5: I find that using a touchpad for controlling TV is fast. & 4.30 & 0.70 \\
\hline PE6: I find that using the system could enhance the quality of life. & 4.23 & 0.86 \\
\hline PE7: I find that using the system would be useful in daily life. & 4.27 & 0.74 \\
\hline
\end{tabular}




\begin{tabular}{lcc}
\hline \multicolumn{1}{c}{ Questionnaire } & Mean & $\begin{array}{c}\text { Standard } \\
\text { Deviation }\end{array}$ \\
\hline Effort Expectancy (EE) & 4.41 & 0.62 \\
\hline EE1: I find that the system is easy to use and not complicated. & 4.63 & 0.61 \\
\hline EE2: I find the system easy to learn and use. & 4.53 & 0.78 \\
\hline EE3: I could learn how to use the system myself. & 4.07 & 0.87 \\
\hline Social Influence (SI) & 4.00 & 0.97 \\
\hline $\begin{array}{l}\text { SI1: I will use the system if my family members encourage and need } \\
\text { me to use it. }\end{array}$ & 3.97 & 1.13 \\
\hline SI2: I will use the system if my family members use it. & 4.10 & 0.92 \\
\hline SI3: I will use the system if people around me use it. & 3.93 & 1.01 \\
\hline Facilitating Conditions (FC) & 3.70 & 0.97 \\
\hline FC1: I have enough necessary resources to use the system. & 3.70 & 1.24 \\
\hline FC2: I have enough necessary knowledge to use the system. & 3.90 & 1.16 \\
\hline $\begin{array}{l}\text { FC3: The system could launch the Argong home screen when the TV } \\
\text { is turned on. }\end{array}$ & 4.10 & 0.88 \\
\hline $\begin{array}{l}\text { FC4: The system could launch the Argong home screen when the } \\
\text { tablet computer is turned on. }\end{array}$ & 4.17 & 0.83 \\
\hline Experience (EXP) & & \\
\hline EXP1: I have experience of how to use a TV. & 3.02 & 0.84 \\
\hline EXP2: I have experience of how to use a TV remote control. & 3.83 & 1.05 \\
\hline EXP3: I have experience of how to use a tablet computer. & 2.37 & 0.75 \\
\hline EXP4: I have experience of how to use a smartphone. & 2.47 & 1.33 \\
\hline EXP5: I have experience of how to use a computer. & 2.27 & 1.43 \\
\hline Voluntariness of Use (VOU) & 4.27 & 1.06 \\
\hline $\begin{array}{l}\text { VOU1: I am willing to use the system myself although no one has } \\
\text { asked me to do so. }\end{array}$ & 4.20 & 1.16 \\
\hline $\begin{array}{l}\text { VOU2: I am willing to use the system myself although it is not } \\
\text { required by any law, rule or regulation. }\end{array}$ & 4.33 & 0.99 \\
\hline Behavioural Intention (BI) & 3.90 & 0.91 \\
\hline BI1: I am willing to use the system. & 3.87 & 0.94 \\
\hline BI2: I expect/intend to use the system in the future. & 0.91 \\
\hline
\end{tabular}

\subsection{Results}

\subsubsection{Results of hypothesis testing}

We used Pearson correlation method for the hypothesis testing as shown in Table 4. The range of the correlation is between -1 and 1 . The negative or positive value is related to behavioural intention, and there is no correlation when the value is 0 . All factors are significant at the significant level $p<0.001$. The detailed results are as follows. Performance Expectancy $(r=0.834, p<0.001)$, Effort Expectancy $(r=0.791, p<0.001)$, Social Influence $(r=0.820, p<0.001)$, Facilitation Conditions $(r=0.599, p<0.001)$, Experience $(r=0.298$, $\mathrm{p}>0.001)$, and Voluntariness $(\mathrm{r}=0.743, \mathrm{p}<0.001)$. The relationships in hypotheses H1, H2, H3, H4, and H6 are significantly strong, while $\mathrm{H} 5$ has no significant influence on the behavioural intention to use the Argong system. 
Table 4. Results of Hypothesis Testing.

\begin{tabular}{llll}
\hline Hypothesis & $\begin{array}{l}\text { Pearson } \\
\text { Correlation (r) }\end{array}$ & Significant (p) & Conclusion \\
\hline H1: $\mathrm{PE}=>$ BI & $0.834^{* *}$ & 0.000 & Accepted \\
H2: EE => BI & $0.791^{* *}$ & 0.000 & Accepted \\
H3: $\mathrm{SI}=>$ BI & $0.820^{* *}$ & 0.000 & Accepted \\
H4: $\mathrm{FC}=>$ BI & $0.599 * *$ & 0.000 & Accepted \\
H5: $\mathrm{EXP}=>$ BI & 0.298 & 0.109 & Not Significant \\
H6: VOU => BI & $0.743^{* *}$ & 0.000 & Accepted \\
\hline
\end{tabular}

Note. ** Correlation is significant at the 0.01 level (2-tailed).

\subsubsection{Limitations}

The limitations of the Argong system are listed as follows:

- The remote control features similar to a traditional TV remote control work with Samsung brand only.

- Tablet computer used as the TV remote control requires a built-in infrared blaster.

- The Android set-top box needs an owner's authorization to access the Android getevent and sendevent commands for a cursor controlling on the TV screen.

\section{Conclusions}

We developed and implemented the Argong multimedia delivery system for the elderly, comprising Argong TV and Argong TV remote services. It was designed to be simple and easy to use by the elderly, with the intention to reduce loneliness and social isolation and increase interactions. The system comprised TV channel, message, reminder, touchpad, and remote control modules. We thereafter studied the factors that affected the multimedia delivery platform based on the UTAUT model, and improved the communication gap between the elderly, family members and friends. The survey instruments were analyzed for the reliability coefficients. The results showed strong relationships of performance expectancy, effort expectancy, social influence, facilitation conditions, and perceived security, while the experience had no significant influence on the behavioural intention to use the Argong application. The findings had important practical implications that could be used as a prototype for improved communication between the elderly, family members and friends. The elderly could effectively send the messages to family members and friends (e.g., sending text messages, images, videos, or sound). In addition, we found that the average performance of using the touchpad as a virtual remote control was better than the traditional remote control, with a faster movement time.

\subsection{Future Work}

Argong system is a native application which is limited to the Android OS devices. This may prevent it from becoming a widely used application for the elderly, in large part due to the wide iOS user community. Nowadays there are various technologies that help developers to implement an application that can be used and installed on various platforms with just one written source code. One of them is Cordova which is a technology from Adobe Corporation and is built to support current technology. The principle of Cordova application is to enable developers to build applications for mobile devices using HTML, CSS and JavaScript, instead of relying on platform specific native application. When packaging and building application in Cordova, the developer has an option to specify which platform to install, such as iOS, Android and Windows. The original developmental intention of the Argong application is to build a web-based application because users can use the application at any place and any time by using any devices with the Internet-connected web browsers. Therefore, Cordova is a suitable tool for developing our improved system with a lower cost and time of developing and maintaining the application, compared with using a platform specific tool for each operating system and device. 


\section{Acknowledgments}

We wish to thank all members of Data Science and Engineering Laboratory (D-Lab), School of Information Technology at King Mongkut's University of Technology Thonburi (KMUTT) for their useful comments, feedback, and technical support. We are grateful to the volunteers who participated in the project, and without them this work would not be completed. We also would like to acknowledge the financial support from the Higher Education Research Promotion and National Research University Project of Thailand, Office of the Higher Education Commission. This work passed the institutional review board (IRB) process of the School of Liberal Arts, KMUT'T, in October 2016.

\section{References}

[1] United Nations, Department of Economic and Social Affairs, Population Division. (2015). World Population Ageing 2015 [Online]. Available: http://www.un.org/en/development/desa/ population/publications/pdf/ageing/WPA2015_Report.pdf [Accessed: 13 June 2017]

[2] Social and Quality of Life Database System. The Aging Population for the Year 1990 to 2040 [Online]. Available: http://social.nesdb.go.th/SocialStat/StatReport_Final.aspx?reportid=1209\&template $=2 \mathrm{R} 1 \mathrm{C} \&$ yeartype $=$ M\&subcatid $=27$ [Accessed: 13 June 2017]

[3] Division-in-Charge. National Statistical Office. The 2014 survey of the older persons in Thailand [Online]. Available: http://service.nso.go.th/nso/nsopublish/themes/files/elderlyworkFullReport57-1.pdf [Accessed: 13 June 2017]

[4] K. Reid, "Lifeline or leisure?: TV's role in the lives of the elderly," Media \& Values, no. 45, 1989. Available: http://www.medialit.org/reading-room/lifeline-or-leisure-tvs-role-lives-elderly [Accessed: 13 June 2017]

[5] J. Choomkasean, P. Mongkolnam, and J. H. Chan, "Multimedia delivery for elderly people: A conceptual model," in International Conference on Advances in Information Technology, Communications in Computer and Information Science, Springer, Berlin, Heidelberg, 2012, vol. 344, pp. 58-69. doi: 10.1007/978-3-642-350764_6

[6] P. Mongkolnam, J. Choomkasean, and J. H. Chan, "Stay in Touch: Multimedia delivery for elderly people," in 2013 International Symposium on Computational and Business Intelligence, New Delhi, India, 2013, pp. 305-309. doi: 10.1109/ISCBI.2013.74

[7] N. Limdumrongnukoon, P. Mongkolnam, T. Visutarrom, and J. H. Chan, "User interface of multimedia delivery platform for the elderly," in The Sixth International Conference on the Applications of Digital Information and Web Technologies, Advances in Digital Technologies, Frontiers in Artificial Intelligence and Applications, IOS press, 2015, vol. 275, pp. 55-67,. doi: 10.3233/978-1-61499-503-6-55

[8] D. Castilla, A. Garcia-Palacios, I. Miralles, J. Breton-Lopez, E. Parra, S. Rodriguez-Berges, and C. Botella, "Effect of Web navigation style in elderly users," Computers in Human Behavior, vol. 55, pp. 909-920, 2016. doi: 10.1016/j.chb.2015.10.034

[9] K. Bozan, B. Davey, and K. Parker, "Social influence on health IT adoption patterns of the elderly: An institutional theory based use behavior approach," Procedia Computer Science, vol. 63, pp. 517-523, 2015. doi: https://doi.org/10.1016/j.procs.2015.08.378

[10] E. Stojmenova, M. Debevc, L. Zebec, and B. Imperl, "Assisted living solutions for the elderly though interactive TV," Multimedia Tools and Applications, vol. 66, no. 1, pp. 115-129, 2013. doi: 10.1007/s11042011-0972-1

[11] V. Orso, A. Spagnolli, L. Gamberini, F. Ibañez, and M. E. Fabregat, "Interactive multimedia content for older adults: the case of Senior Channel," Multimedia Tools and Applications, vol. 76, no. 4, pp. 5171 5189, 2016. doi: 10.1007/s11042-016-3553-5

[12] S. C. Wang, T. C. Chung, and K. Q. Yan, "A new territory of multi-user variable remote control for interactive TV," Multimedia Tools and Applications, vol. 51, no. 3, pp. 1013-1034, 2011. doi: 10.1007/s11042-009-0435-0

[13] T. Rodriguez, K. Fischer, and J. Kingston, "Intelligent services for the elderly over the TV," Journal of Intelligent Information Systems, vol. 25, no. 2, pp. 159-180, 2005. doi: 10.1007/s10844-005-0187-x

[14] S. Spinsante and E. Gambi, "Remote health monitoring for elderly through interactive television," Biomedical Engineering Online, p. 54, 2012. doi: 10.1186/1475-925X-11-54 
[15] C. Bae, J. Yoo, K. Kang, Y. Choe, and J. Lee, "Home server for home digital service environments," IEEE Transactions on Consumer electronics, pp. 382-383, 2003. doi: 10.1109/ICCE.2003.1218984

[16] M. Nikolova, F. Meijs, and P. Voorwinden, "Remote mobile control of home appliances," IEEE Transactions on Consumer Electronics, vol. 49, no. 1, pp. 123-127, 2003. doi: 10.1109/TCE.2003.1205464

[17] V. Lopes, E. Queiroz, N. Freitas, M. Oliveira, and O. Monteiro, "TV-Health: A context-aware health care application for Brazilian digital TV," in Proceedings of the 22nd Brazilian Symposium on Multimedia and the Web, 2016, pp. 103-106. doi: 10.1145/2976796.2988170

[18] 8 Great Apps For Our Elders [Online]. Available: https://www.forbes.com/sites/nextavenue/2015/ 08/28/8-great-apps-for-our-elders [Accessed: 13 June 2017]

[19] Y. Xiao, X. Du, and J. Zhang, "Internet protocol television (IPTV): The Killer application for the nextgeneration Internet," IEEE Communications Magazine, vol. 45, no. 11, pp. 126-134, 2007. doi: 10.1109/MCOM.2007.4378332

[20] Android Developers. RecognizerIntent [Online]. Available: https://developer.android.com/reference/ android/speech/RecognizerIntent.html [Accessed: 13 June 2017]

[21] Y. R. Lai and T. P. Hwang, "Virtual touchpad for cursor control of touchscreen thumb operation in the mobile context," International Conference on Design, User Experience and Usability, vol. 9187, pp. 563-574, 2015. doi: 10.1007/978-3-319-20898-5_54

[22] Gitiles. Getevent [Online]. Available: http://source.android.com/devices/input/getevent.html [Accessed: 13 June 2017]

[23] Gitiles. getevent.c [Online]. Available: https://android.googlesource.com/platform/system/core/+/ android-4.4_r1/toolbox/getevent.c [Accessed: 13 June 2017]

[24] Gitiles. sendevent.c [Online]. Available: https://android.googlesource.com/platform/system/ core/+/android-4.4_r1/toolbox/sendevent.c [Accessed: 13 June 2017]

[25] Android Developers. MotionEvent [Online]. Available: https://developer.android.com/reference/ android/view/MotionEvent.html [Accessed: 13 June 2017]

[26] K. K. Cheong, I. Kim, S. K. Park, and Y. J. Park, "User performance measures for evaluating interactive TV pointing devices," IEEE Transactions on Consumer Electronics, vol. 57, no. 3, pp. 1236-1244, 2011. doi: 10.1109/TCE.2011.6018879

[27] R. Senanayake, R. S. Goonetilleke, and E. R. Hoffmann, "Targeted-tracking with pointing devices," IEEE Transactions on Human-Macbine Systems, vol. 45, no. 4, pp. 431-441, 2015. doi: 10.1109/THMS.2015.2408260

[28] I. S. MacKenzie, "Fitts' throughput and the remarkable case of touch-based target selection," in International Conference on Human-Computer Interaction, 2015, pp. 238-249. doi: 10.1007/978-3-319-209166_23

[29] O. Chapuis, R. Blanch, and M. Beaudouin-Lafon. (2017). Fitts' Law in the Wild: A Field Study of Aimed Movements [Online]. Available: https://hal.archives-ouvertes.fr/hal-00612026

[30] S. MacKenzie. Fitts' Law Software Download [Online]. Available: http://www.yorku.ca/mack/ FittsLawSoftware [Accessed: 13 June 2017]

[31] International Organization for Standardization, ISO 9241-9:2000 [Online]. Available: http://www.iso.org/iso/catalogue_detail.htm?csnumber=30030 [Accessed: 13 June 2017]

[32] Android Developers. ConsumerIrManager [Online]. Available: https://developer.android.com/ reference/android/hardware/ConsumerIrManager.html [Accessed: 13 June 2017]

[33] V. Venkatesh, M. G. Morris, G. B. Davis, and F. D. Davis, "User acceptance of information technology: Toward a unified view," MIS Quarterly, vol. 27, no. 3, pp. 425-478, 2003.

[34] M. Cimperman, M. M. Brenčič, and P. Trkman, "Analyzing older users' home telehealth services acceptance behavior-Applying an Extended UTAUT model," International Journal of Medical Informatics, vol. 90, pp. 22-31, 2016. doi: 10.1016/j.ijmedinf.2016.03.002

[35] V. Techatraiphum, A. Tharnuraikun, W. Krathu, and W. Chutimaskul, "Telemedicine acceptance framework for the elderly in Thailand," in 2016 International Conference on Information and Communication Technology Convergence (ICTC), Jeju, pp. 39-44, 2016. doi: 10.1109/ICTC.2016.7763430

[36] S. N. Attuquayefio, and H. Addo, "Using the UTAUT model to analyze students' ICT adoption," International Journal of Education and Development using Information and Communication Technology, vol. 10, no. 3, p. 75, 2014.

[37] C. Kufs. (2010). 30 Samples. Standard, Suggestion, or Superstition? [Online]. Available: http://statswithcats.wordpress.com/2010/07/11/30-samples-standard-suggestion-or-superstition [Accessed: 13 June 2017] 
[38] L. J. Cronbach and R. J. Shavelson, "My current thoughts on coefficient alpha and successor procedures," Educational and Psychological Measurement, vol. 64, no. 3, pp. 391-418, 2004. doi: 10.1177/0013164404266386 